\title{
Response of Post-Mastectomy Lymphedema to Extracorporeal Shockwave Therapy
}

\author{
Mahmoud El-Shazly ${ }^{1}$, Wafaa Hussien Borhan ${ }^{1}$, Wael Naeem Thabet ${ }^{2}$, Ashraaf Hassan ${ }^{1}$ \\ ${ }^{1}$ Department of Physical Therapy for Surgery, Faculty of Physical Therapy, Cairo University, Cairo, Egypt \\ ${ }^{2}$ Department of General Surgery, Faculty of Medicine, Cairo University, Cairo, Egypt
}

Email address:

Mahmoud_elshazly311@yahoo.com (M. El-Shazly),Dr.shazly88@gmail.com (A. Hassan)

\section{To cite this article:}

Mahmoud El-Shazly, Wafaa Hussien Borhan, Wael Naeem Thabet, Ashraaf Hassan. Response of Post-Mastectomy Lymphedema to Extracrorporeal Shockwave Therapy. Journal of Surgery. Special Issue: Surgical Infections and Sepsis. Vol. 4, No. 3-1, 2016, pp. 14-20. doi: $10.11648 /$ j.js.s.2016040301.13

Received: January 31, 2016; Accepted: February 1, 2016; Published: March 23, 2016

\begin{abstract}
Introduction: Post-mastectomy lymphedema is a chronic, debilitating disorder that is frequently misdiagnosed, treated too late or not treated at all, Lymphedema (LE) is an abnormal accumulation of protein-rich fluid in the interstitium leading to limb swelling, chronic inflammation and reactive fibrosis of the affected tissues resulting from damage to lymphatic circulation following surgery, chemotherapy and/or radiation therapy. The aim was to investigate the effect of shockwave therapy on the post-mastectomy lymphedema. Subjects and methods: Sixty female patients underwent modified radical mastectomy surgery or lumpectomy, chemotherapy and radiotherapy associated with unilateral lymphedema (stage 2, 3), into two equal groups; their ages ranged from 30-50 years. The study group received shockwave therapy 2 times/week for 6 weeks plus traditional physical therapy program (manual lymphatic drainage, circulatory exercises with elevation, shoulder ROM exercises and pneumatic compression therapy) 3times /week /6weeks. Control group received traditional physical therapy program (manual lymphatic drainage, circulatory exercises with elevation, shoulder ROM exercises and pneumatic compression therapy) 3times /week /6weeks. Evaluation procedures were carried out to measure the upper limb volume measurement, ROM of shoulder flexion, abduction and external rotation. Results: Post treatment results showed that there was a significant improvement difference in shoulder ROM and upper limb volume in both groups in favor of the study group. Percentage of improvement of shoulder abduction, flexion, and external rotation in the study group was $38.92 \%, 26.61 \%$, $36.24 \%$ respectively, while it was $20.86 \%, 14.93 \%, 16.17 \%$ in the control group. Percentage of improvement of upper limb volume in the study group was $24.21 \%$, while it was $15.5 \%$ in the control group. Conclusion: extracorporeal shockwave therapy was considered to be an effective modality for treatment of the post-mastectomy lymphedema.
\end{abstract}

Keywords: Lymphedema, Mastectomy, Shockwave Therapy

\section{Introduction}

In Egypt, breast cancer is estimated to be the most common cancer among females accounting for $37.7 \%$ of their total with 12,621 new cases in 2008 [1] and secondary lymphedema associated with breast cancer as a result of axillary lymph nodes damage due to radical mastectomy, chemotherapy, and radiotherapy [2]. Lymphedema is a chronic and progressive condition characterized by the excessive, regional interstitial accumulation of protein-rich fluid, chronic inflammation, and fibrosis resulting from an abnormality or damage to the lymphatic system. Secondary lymphedema is caused by multiple factors related with lymphatic stasis, such as tumor lymph node infiltration, lymph node dissection, chemotherapy, radiotherapy, trauma, and infection [3]. Breast cancer related lymphedema can lead to a feeling of heaviness, discomfort, weakness, pain, and restricted shoulder mobility in the involved extremity [4-5]. Range of motion restriction with is a result of tissue manipulation and positioning during surgery also lymphedema, by itself, the sheer weight of the arm further limits movement, can negatively alter posture, and can reduce the functional abilities necessary for independent activities [6-7]. There are different methods to evaluate upper limb volume as water displacement method and arm 
circumference measurements [8].

Second and third stages of lymphedema are characterized by fibrosis (non-pitting edema) due to secondary proliferation of neutrophils, macrophages, and fibroblasts and accumulation of collagen [9]. One of the most common forms of the treatment is complex decongestive physical therapy (CDPT) [10-11]. CDPT involves various techniques such as scrupulous skin care, manual lymphatic drainage, external compression device, exercise to improve lymphatic movement. But the effect of CDPT in the second and third stages of lymphedema is limited due to dermatofibrotic changes [12]. Extracorporeal shockwave (ESWT) has a wide uses in orthopedic cases where it has a positive effect on plantar fasciitis, elbow epicondylitis. Several studies have demonstrated the effect of shockwave on the angiogenesis and suppression of the inflammation [13]. ESWT stimulates the lymph-angiogenesis by regulation the vascular endothelial growth factor and basic fibroblast growth factor [14].

Only pilot studies applied on humans to prove the effect of ESWT on the lymph-angiogenesis and decrease the inflammation and other studies applied on animals to support this idea. The aim of this study was to investigate the effect of ESWT on stage 2, 3 lymphedema.

\section{Subjects, Materials and Methods}

This study was carried out on 60 adult female patients subjected to modified radical mastectomy or lumpectomy with chemotherapy and radiotherapy associated with unilateral lymphedema (stage 2, 3), their ages ranged from 30 to 50 years and selected from the Out Patient Clinic of National Cancer Institute, Cairo Egypt and Faculty of Physical Therapy - Cairo University, Cairo Egypt during the period April 2015 to September 2015. Patients were randomly assigned into two equal groups; Group A (Study group) and Group B (Control group).Group A received shockwave therapy 2 times/week for 6 weeks plus traditional physical therapy program for 60 minutes / 3times / week / 6weeks (included; manual lymphatic drainage for 15 minutes followed by circulatory exercises with elevation for 15 minutes to increase lymphatic drainage then shoulder ROM exercises for 15 and pneumatic compression therapy for 15 minutes) and group B received traditional physical therapy program for 60 minutes / 3times / week / 6weeks.

Patients with the following conditions were excluded from the study; subjects had history of severe trauma or disruptive surgery to the arm, current metastasis, renal, heart, or lung disorders, anomaly of vessel, infection, pregnancy, breast feeding, hyperventilation, polyneuropathy and patients with severe lymphedema (difference arm circumference more than $8 \mathrm{~cm}$ ).

The work has been carried out in accordance with the ethics of committee for experiments at Faculty of Physical Therapy, Cairo University involving humans, and parents filled approval consent to share in the study.

The assessment approaches were: Arm volume measurement, ROM of shoulder flexion, abduction and external rotation were measured with a standard goniometer.

Preparation of shockwave device: it was adjusted with 2000 impulses for $10 \mathrm{~min} .1000$ impulse on the most fibrotic point and 1000 impulse on the lesser fibrotic point, The intensity of treatment was $0.040-0.069 \mathrm{~mJ} / \mathrm{mm} 2$ energy flux density and the frequency was $5 \mathrm{~Hz}$.

\section{Statistical Analysis}

Descriptive statistics and t-test were conducted for comparison of the mean age of both groups. T-test was conducted for comparison of upper limb volume and shoulder ROM between groups. Paired $t$ test was for comparison between pre and post treatment mean values of upper limb volume and shoulder ROM in each group. The level of significance for all statistical tests was set at $p<0.05$. All statistical measures were performed through the statistical package for social studies (SPSS) version 19 for windows.

\section{Results}

The statistical analysis for patient's socio-demographic data (age, sex) revealed a non-significant difference between both groups. There was no significant difference in mean values of shoulder abduction ROM between both groups pretreatment $(p=0.66)$. While there was a significant increase in shoulder abduction ROM in group A post treatment compared to that of group $B(p=0.0001)$ as shown in table 1 and figure 1.

There was no significant difference in mean values of shoulder flexion ROM between both groups pretreatment $(\mathrm{p}=$ 0.39 ). While there was a significant increase in shoulder flexion ROM in group A post treatment compared to that of group B (p $=0.01$ ) as shown in table 2 and figure 2 . Accordingly, there was no significant difference in the mean values of shoulder external rotation ROM pretreatment between group A and B $(p=0.29)$ and a significant increase in shoulder external rotation ROM of group A compared with group B post treatment $(p=0.0001)$ as shown in table 3 and figure 3.

The authors found no significant difference in the mean values of upper limb volume pretreatment between group A and B $(p=0.46)$ and a significant decrease in upper limb volume of group A compared with group B post treatment ( $p$ $=0.0001)$ as shown in table 4 and figure 4 .

Table 1. Pre and post treatment results of shoulder abduction ROM for both groups.

\begin{tabular}{llll}
\hline & Pre treatment & & Post-treatment \\
\cline { 2 - 4 } & Group A & Group B & Group A \\
\hline $\bar{C}$ & 82.13 & 80.8 & 114.1 \\
$\mathrm{SD} \pm$ & \pm 11.2 & \pm 12.38 & 97.66 \\
$\mathrm{t}-$ value & 0.43 & & \pm 14.62 \\
p- value & 0.66 & 4.19 & 0.0001 \\
Significance & Non-significant & & Significant \\
\hline
\end{tabular}


Table 2. Pre and post treatment results of shoulder flexion ROM for both groups.

\begin{tabular}{lllll}
\hline & Pre treatment & & Post-treatment & \\
\cline { 2 - 5 } & Group A & Group B & Group A & Group B \\
\hline $\bar{C}$ & 95.12 & 97.3 & 120.44 & 111.83 \\
$\mathrm{SD} \pm$ & \pm 7.81 & \pm 11.6 & \pm 13.07 & \pm 13.91 \\
t- value & -0.85 & & 2.47 & \\
p- value & 0.39 & Significant & \\
Significance & Non-significant & & . & \\
\hline
\end{tabular}

Table 3. Pre and post treatment results of shoulder external rotation ROM for both groups.

\begin{tabular}{lllll}
\hline & Pre treatment & & Post-treatment & \\
\cline { 2 - 5 } & Group A & Group B & Group A & Group B \\
\hline $\bar{C}$ & 31.51 & 30.3 & 42.93 & 35.2 \\
$\mathrm{SD} \pm$ & \pm 3.68 & \pm 5.12 & \pm 5.04 & \pm 4.62 \\
$\mathrm{t}-$ value & 1.05 & & 6.18 & \\
p- value & 0.29 & 0.0001 & \\
Significance & Non-significant & & Significant & \\
\hline
\end{tabular}

Table 4. Pre and post treatment results of upper limb volume for both groups.

\begin{tabular}{lllll}
\hline & Pre treatment & & Post-treatment & Group B \\
\cline { 2 - 5 } & Group A & Group B & Group A & 1043.85 \\
$\bar{C}$ & 1219.33 & 1235.4 & 924.04 & \pm 90.32 \\
$\mathrm{SD} \pm$ & \pm 83.42 & \pm 84.12 & \pm 94.71 & -5.01 \\
$\mathrm{t}-$ value & -0.74 & & 0.0001 & \\
p- value & 0.46 & Significant & \\
Significance & Non-significant & & & \\
\hline
\end{tabular}

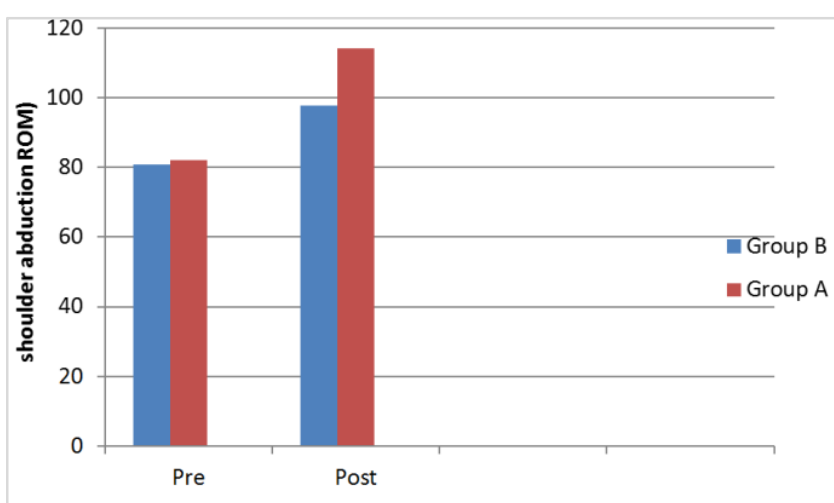

Figure 1. Pre and Post-treatment mean values of shoulder abduction ROM for both groups.

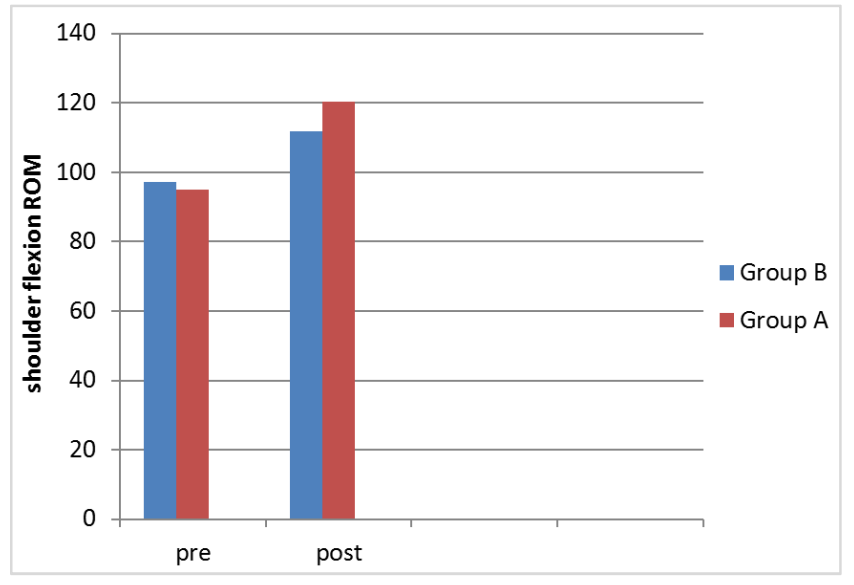

Figure 2. Pre and Post-treatment mean values of shoulder flexion ROM for both groups.

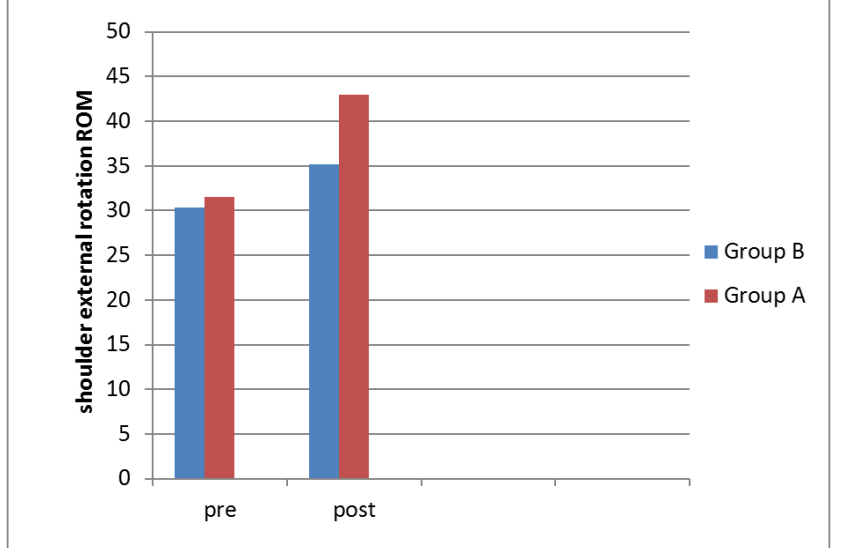

Figure 3. Pre and Post-treatment mean values of shoulder external rotation for both groups.

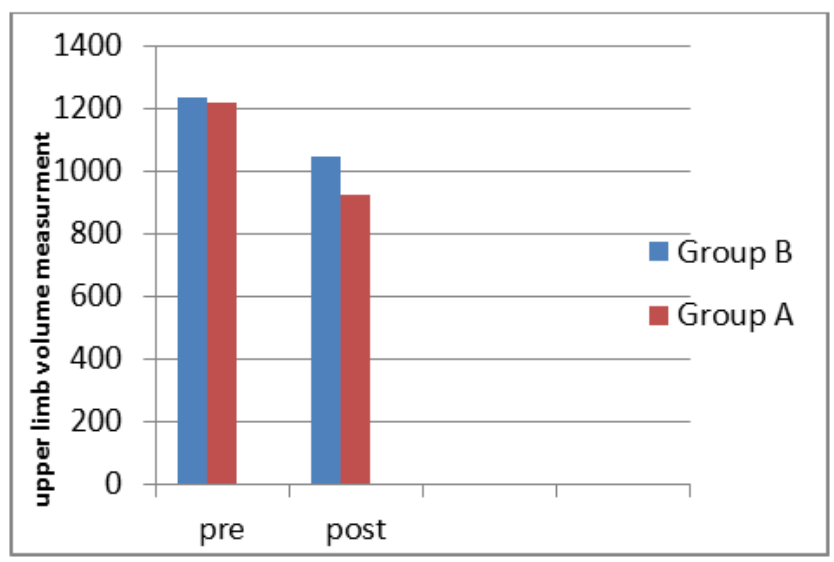

Figure 4. Pre and Post-treatment mean values of upper limb volume for both groups. 
Statistical analysis of results showed a significant improvement in shoulder abduction, flexion, external rotation and upper limb volume in group A and group B after 6 weeks of treatment as follows:

Result of shoulder abduction ROM: The mean \pm SD pretreatment of group A was $82.13 \pm 11.2$ degrees and that post treatment was $114.1 \pm 14.62$ degrees. The mean difference between pre and post treatment was -31.97 degrees and the percent of improvement was $38.97 \%$. There was a significant increase in shoulder abduction ROM of group A post treatment compared with pretreatment $(\mathrm{p}=$ 0.0001). while in group B The mean \pm SD shoulder abduction ROM pretreatment was $80.8 \pm 12.38$ degrees and that post treatment was $97.66 \pm 15.7$ degrees. The mean difference between pre and post treatment was -16.86 degrees and the percent of improvement was $20.86 \%$. There was a significant increase in shoulder abduction ROM of group B post treatment compared with pretreatment $(\mathrm{p}=$ 0.0001 ) as shown in table 5 and figure 5.

Result of shoulder flexion ROM: The mean \pm SD pretreatment of group A was $95.12 \pm 7.81$ degrees and that post treatment was $120.44 \pm 13.07$ degrees. The mean difference between pre and post treatment was -25.32 degrees and the percent of improvement was $26.61 \%$. There was a significant increase in shoulder flexion ROM of group A post treatment compared with pretreatment $(p=0.0001)$. while in group $\mathrm{B}$ The mean $\pm \mathrm{SD}$ shoulder flexion $\mathrm{ROM}$ pretreatment was $97.3 \pm 11.6$ degrees and that post treatment was $111.83 \pm 13.91$ degrees. The mean difference between pre and post treatment was -14.53 degrees and the percent of improvement was $14.93 \%$. There was a significant increase in shoulder flexion ROM of group B post treatment compared with pretreatment $(p=0.0001)$ as shown in table 6 figure 6.

Result of shoulder external rotation ROM: The mean \pm SD shoulder external rotation ROM pretreatment of group A was $31.51 \pm 3.68$ degrees and that post treatment was $42.93 \pm$ 5.04 degrees. The mean difference between pre and post treatment was -11.42 degrees and the percent of improvement was $36.24 \%$. There was a significant increase in shoulder external rotation ROM of group A post treatment compared with pretreatment $(\mathrm{p}=0.0001)$. While in group $\mathrm{B}$ The mean \pm SD shoulder external rotation ROM pretreatment was $30.3 \pm 5.12$ degrees and that post treatment was $35.2 \pm$ 4.62 degrees. The mean difference between pre and post treatment was -4.9 degrees and the percent of improvement was $16.17 \%$. There was a significant increase in shoulder external rotation ROM of group B post treatment compared with pretreatment $(\mathrm{p}=0.0001)$ as shown in table 7 figure 7 .

Result of upper limb volume measurement: The mean \pm SD upper limb volume pretreatment of group A was 1219.33 $\pm 83.42 \mathrm{ml}$ and that post treatment was $924.04 \pm 94.71 \mathrm{ml}$. The mean difference between pre and post treatment was $295.29 \mathrm{ml}$ and the percent of improvement was $24.21 \%$. There was a significant decrease in upper limb volume of group A post treatment compared with pretreatment $(\mathrm{p}=$ $0.0001)$. While in group B the mean \pm SD upper limb volume pretreatment was $1235.4 \pm 84.12 \mathrm{ml}$ and that post treatment was $1043.85 \pm 90.32 \mathrm{ml}$. The mean difference between pre and post treatment was $191.55 \mathrm{ml}$ and the percent of improvement was $15.5 \%$. There was a significant decrease in upper limb volume of group B post treatment compared with pretreatment $(\mathrm{p}=0.0001)$ as shown in table 8 figure 8 .

Table 5. Pre and post treatment results of shoulder abduction ROM for each group.

\begin{tabular}{|c|c|c|c|c|}
\hline & Group A & & Group B & \\
\hline & Pre & Post & Pre & Post \\
\hline$\overline{\mathrm{C}}$ & 82.13 & 114.1 & 80.8 & 97.66 \\
\hline $\begin{array}{l}\mathrm{SD} \pm \\
\text { t- value } \\
\text { p- value } \\
\text { Significance }\end{array}$ & $\begin{array}{l}11.2 \pm \\
-17.88 \\
0.0001 \\
\text { Significant }\end{array}$ & $14.62 \pm$ & $\begin{array}{l}12.38 \pm \\
-8.79 \\
0.0001 \\
\text { Significant }\end{array}$ & $15.7 \pm$ \\
\hline
\end{tabular}

Table 6. Pre and post treatment results of shoulder flexion ROM for each group.

\begin{tabular}{|c|c|c|c|c|}
\hline & Group A & & Group B & \\
\hline & Pre & Post & Pre & Post \\
\hline$\overline{\mathrm{C}}$ & 95.12 & 120.44 & 97.3 & 111.83 \\
\hline $\begin{array}{l}\mathrm{SD} \pm \\
\text { t- value } \\
\text { p- value } \\
\text { Significance }\end{array}$ & $\begin{array}{l} \pm 7.81 \\
-17.88 \\
0.0001 \\
\text { Significant }\end{array}$ & \pm 13.07 & $\begin{array}{l} \pm 11.6 \\
-13.16 \\
0.0001 \\
\text { Significant }\end{array}$ & \pm 13.91 \\
\hline
\end{tabular}

Table 7. Pre and post treatment results of shoulder external rotation ROM for each group.

\begin{tabular}{|c|c|c|c|c|}
\hline & Group A & & Group B & \\
\hline & Pre & Post & Pre & Post \\
\hline$\overline{\mathrm{C}}$ & 31.51 & 42.93 & 30.3 & 35.2 \\
\hline $\mathrm{SD} \pm$ & \pm 3.68 & \pm 5.04 & \pm 5.12 & \pm 4.62 \\
\hline t- value & -25.48 & & -13.16 & \\
\hline p- value & 0.0001 & & 0.0001 & \\
\hline Significance & Significant & & Significant & \\
\hline
\end{tabular}


Table 8. Pre and post treatment results of upper limb volume for each group.

\begin{tabular}{lllll}
\hline & Group A & & Group B & \\
\cline { 2 - 5 } & Pre & Post & Pre & Post \\
\hline $\bar{C}$ & 1219.33 & 924.04 & 1235.4 & 1043.85 \\
$\mathrm{SD} \pm$ & \pm 83.42 & \pm 94.71 & \pm 84.12 & \pm 90.32 \\
$\mathrm{t}-$ value & 20.92 & & 19.36 & \\
$\mathrm{p}$ - value & 0.0001 & & 0.0001 & \\
Significance & Significant & & Significant & \\
\hline
\end{tabular}

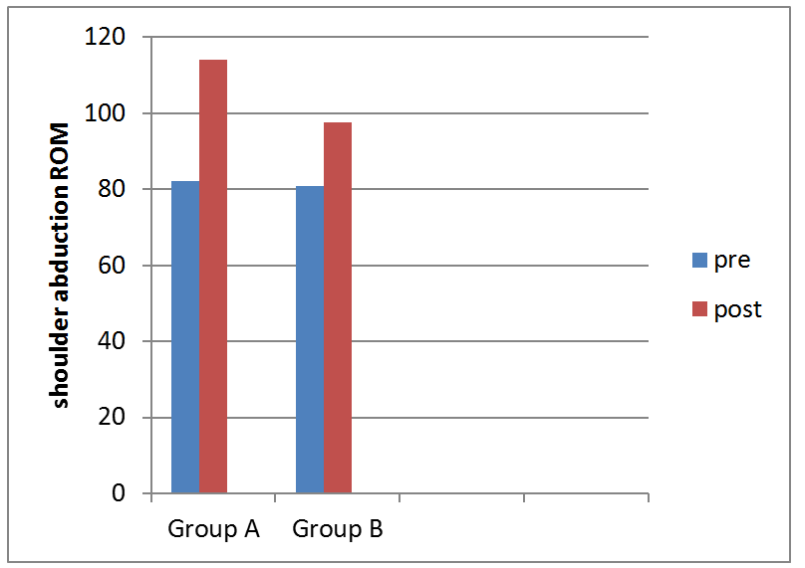

Figure 5. Mean values of pre and post treatment of shoulder abduction ROM for each group.

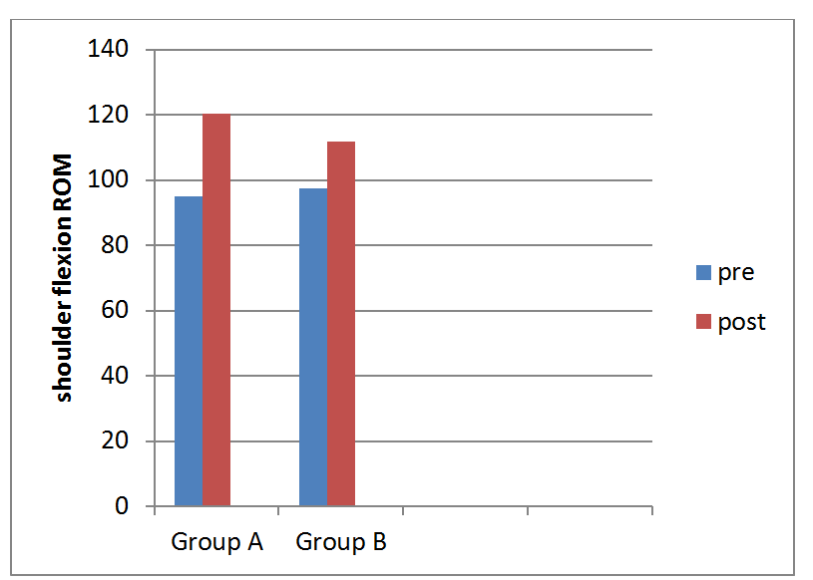

Figure 6. Mean values of pre and post treatment of shoulder flexion ROM for each group.

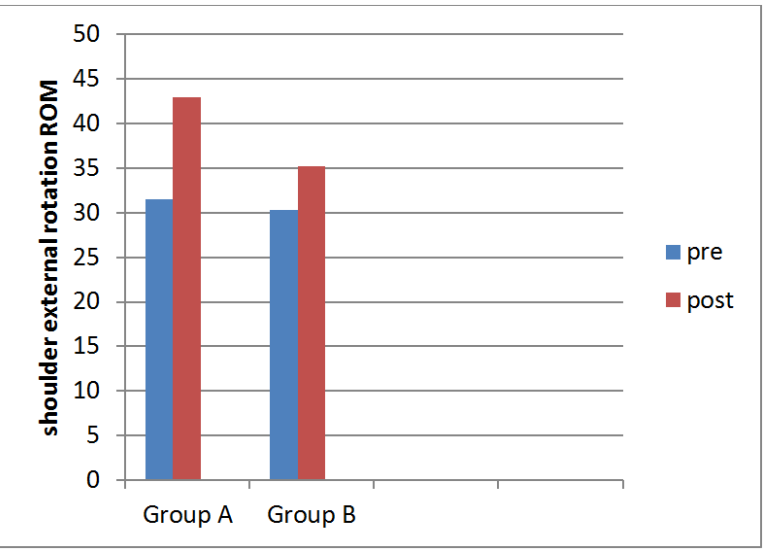

Figure 7. Mean values of pre and post treatment of shoulder external rotation for each group.

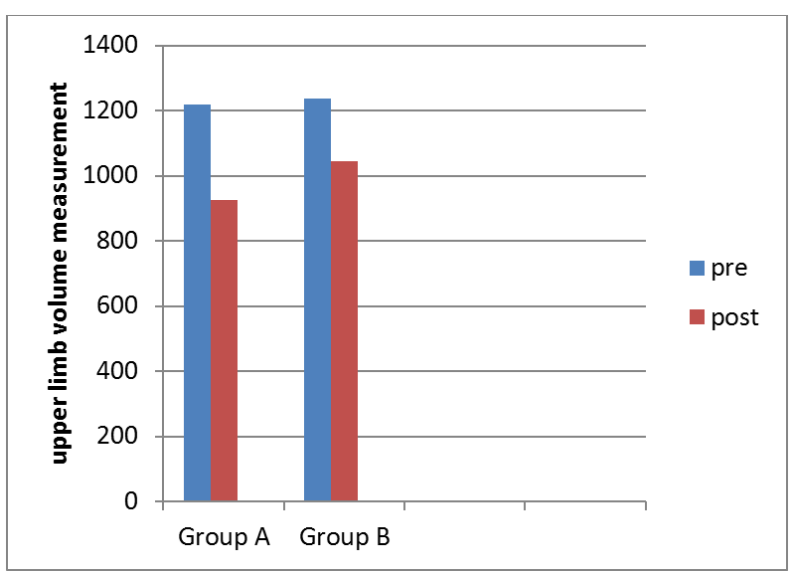

Figure 8. Mean values of pre and post treatment of upper limb volume for each group.

\section{Discussion}

The findings of this study indicated that patients suffering from upper limb lymphedema following surgery of mastectomy had a significant improvement in shoulder abduction, flexion, external rotation and upper limb volume after application of shockwave therapy plus traditional physical therapy. The statistical analysis revealed a significant improvement of shoulder abduction ROM in the study group than that of the control group; the percentage of improvement was $38.92 \%$ and $20.86 \%$ for the two groups respectively. Also there is a significant improvement of shoulder flexion ROM in the study group than that of the control group; the percentage of improvement was $26.61 \%$ and $14.93 \%$ for the two groups respectively. Also there is a significant improvement of shoulder external rotation ROM in the study group than that of the control group; the percentage of improvement was $36.24 \%$ and $16.17 \%$ for the two groups respectively.

Also there is a significant improvement of upper limb volume measurement in the study group than that of the control group; the percentage of improvement was $24.21 \%$ and $15.5 \%$ for the two groups respectively. These results are consistent with authors who supported that shockwave therapy is an effective modality in treatment of upper limb lymphedema after mastectomy surgery as follows:

After application of shockwave therapy for treatment of lymphedema there was a medium decreasing of the $32 \%$ of the circumference of the limb and a subjective and objective reduction of tissural consistence, above all in corresponding fibrotic areas. [15]

Shockwave therapy improved the condition of the 
lymphedema-affected limbs of post-menopausal patients, as assessed by a number of criteria. The mean affected limb volume as well as mean of total circumferential arm difference demonstrated a trend toward reduction over time of study, and was better than control group 4wks post treatment and also was high significantly better than control group $8 \mathrm{wks}$ post treatment. As a sequence effect of lymphedema, limited ROM demonstrated a trend toward improvement over time of study and was better than control group 4wks post treatment and also was high significantly better than control group $8 \mathrm{wks}$ post treatment. [16]

72 patients affected by primary (25) of secondary (47) lymphedema of upper and lower limbs treated with shockwave therapy for 10 sessions. Patients were evaluated 2 weeks and 1 month after treatment. They show an average decrease of the $26 \%$ of the circumference of the limb and a subjective and objective reduction of tissue consistence, above all in fibrotic areas. So shock wave therapy is very useful in primary and secondary lymphedema, not only for the decreasing of the limb volume, but above all for the treatment of the fibrotic areas. [17]

A pilot study applied on 7 patients which evaluated the effectiveness of ESWT as a treatment technique for the management of stage 3 secondary lymphedema by using Clinical measurements such as the volume and circumference of the upper limb and skin fold thickness were changed between before and after treatment. All patients treated with 4 sessions of shockwave therapy and showed significant volume reduction (37.23\%). [12]

Low-energy ESWT induces therapeutic lymphangiogenesis by up-regulating vascular endothelial growth factor $\mathrm{C}$ and basis fibroblast growth factor, and by improving lymphedema in a rat model. [18] So we demonstrate that improvement of post-mastectomy lymphedema occurred due to lymphangiogenesis. Serizawa et al. created a rat tail model of lymphedema and subsequently subjected the animals to serial ECT therapy. Enhanced drainage of lymphaticfluid as well as up-regulation of VEGF-C expression was found in the treatment group compared to the controls. [18]

Study was designed to employ established methods to analyze shockwave-induced lymphangiogenesis in vitro. We show that shockwaves alter the biological properties of LEC in terms of proliferation, migration, morphology, marker profiles and gene expression. [19]

From the gained results it could be concluded that treatment of post-mastectomy lymphedema with shockwave therapy is an effective. Therefore, this noninvasive treatment provides clinically favorable outcome to patients with breast cancer-related lymphedema.

\section{References}

[1] Aly El-Din N. Cancer statistics 2002-2007. Preliminary report [online]. Available at: $<$ http://www.nci.cu.edu.eg/lectures/Cancer_statistics20022007.pdf $>$; 2010 (accessed 05.09.2012).
[2] So-Yeon Kim, MD, HasukBae, MD, Hye Min Ji, MD). A case report of 'Computed Tomography as an Objective Measurement Tool for Secondary Lymphedema Treated With Extracorporeal Shock Wave Therapy'. Ann Rehabil Med 2015; 39(3): 488-493.

[3] Warren AG, Brorson H, Borud LJ, Slavin SA. Lymphedema: a comprehensive review. Ann PlastSurg 2007; 59: 464-72.

[4] Harris SR, et al.: "Clinical guidelines for the care and treatment of breast cancer: II Lymphedema", 2001, CMAI; 23: 1 .

[5] Rietman JS, et al., "Long term treatment related upper limb morbidity and quality of life after sentinel lymph node biopsy for stage I or II breast cancer. Eur. J. Surg. Oncol. 2006; 32: 148 .

[6] Brennan MJ, De Pompolo RW, and Garden FH, "Focused review: Postmastectomy lymphedema" Arch. Phys. Med. Rehabil; 1996, 77(Suppl): S74.

[7] Warmuth MA, et al.: "Complications of axillary lymph node dissection for carcinoma of the breast", Cancer; 1998, 83: 1362 .

[8] Richard Taylor, Upali W Jayasinghe, Louise Koelmeyer, Owen Ung and John Boyages. 'Reliability and Validity of Arm Volume Measurements for Assessment of Lymphedema'. Journal Of The American Physical Therapy Association February 2006 vol. 86 no. 2 205-214.

[9] Korpan MI, Crevenna R, Fialka-Moser V. Lymphedema: a therapeutic approach in the treatment and rehabilitation of cancer patients. Am J Phys Med Rehabil 2011; 90 (5 Suppl 1): S69-75.

[10] Casley-Smith JR, Casley-Smith JR. Treatment of lymphedema by complex physical therapy, with and without oral and topical benzopyrones: what should therapists and patients expect. Lymphology 1996; 29: 76-82.

[11] Williams AF, Vadgama A, Franks PJ, Mortimer PS. A randomized controlled crossover study of manual lymphatic drainage therapy in women with breast cancer-related lymphoedema. Eur J Cancer Care (Engl) 2002; 11: 254-61.

[12] HasukBae, MD, Ho Jeong Kim, MD Department of Rehabilitation Medicine, Ewha Womans University School of Medicine, Seoul, Korea. Clinical Outcomes of Extracorporeal Shock Wave Therapy in Patients with Secondary Lymphedema: A Pilot Study. Ann Rehabil Med 2013; 37(2): 229-234.

[13] Schaden W, Thiele R, Kolpl C, Pusch M, Nissan A, Attinger $\mathrm{CE}$, et al. Shock wave therapy for acute and chronic soft tissue wounds: a feasibility study. J Surg Res 2007; 143: 1-12.

[14] Serizawa F, Ito K, Matsubara M, Sato A, Shimokawa H, Satomi S. Extracorporeal shock wave therapy induces therapeutic lymphangiogenesis in a rat model of secondary lymphoedema. Eur J VascEndovascSurg 2011; 42: 254-60.

[15] Michelini S, Failla A, Moneta G, Zinicola V, Macaluso B, Cardone M, Antonucci D: Treatment of Lymphedema with shockwave therapy: preliminary study. The European Journal of Lymphologyand related problems. 2007, 17(51): 29.

[16] Hesham G Mahran, Ali A Thabet: "Extracorporeal Shockwave Therapy for Post-Menopausal Patients with Breast CancerRelated Lymphedema" International Journal of Cancer Research ISSN: 2015, 2051-784X, Vol. 49, Issue. 1. 
[17] Michelini et al.: "Treatment of geriatrics lymphedema with shockwave therapy" BMC Geriatrics, 2010, 10 (Suppl 1): A105.

[18] Serizawa, F., Ito, K., Matsubara, M., Sato, A., Shimokawa, H., \& Satomi, S: Extracorporeal shock wave therapy induces therapeutic lymphangiogenesis in a rat model of secondary lymphoedema. European Journal of Vascular and Endovascular Surgery, 2011, Vol. 42, No. 2, pp. 254-260.
[19] Rohringer S, Holnthoner W, Hackl M, Weihs AM, Rünzler D, et al.: Molecular and Cellular Effects of In Vitro Shockwave Treatment on Lymphatic Endothelial Cells. PLoS ONE, 2014, 9(12): e114806. doi: 10.1371/journal.pone.0114806. 